\title{
Consensus Guidelines for the Diagnosis and Treatment of Growth Hormone (GH) Deficiency in Childhood and Adolescence: Summary Statement of the GH Research Society*
}

\author{
GH RESEARCH SOCIETY†
}

Medical Department M, Aarhus Kommunehospital, DK-8000, Aarhus C, Denmark

Received March 20, 2000. Revision received July 11, 2000. Accepted July 12, 2000.

Address all correspondence and requests for reprints to: GRS Secretariat, Medical Department M, Aarhus Kommunehospital, DK-8000, Aarhus C, Denmark.

* These consensus guidelines have been endorsed by the following international societies: the Councils and Drug and Therapeutics Committees of the European Society for Pediatric Endocrinology and the Lawson Wilkins Pediatric Endocrine Society. It has also been endorsed by the Councils of the Australasian Pediatric Endocrinology Group, the Japanese Society for Pediatric Endocrinology, and the Sociedad Latinoamericana de Endocrinologia Pediatrica.

t List of participants in the Growth Hormone Research Society Workshop on Child and Adolescent Growth Hormone Deficiency, Eilat, Israel, October 17-21, 1999: Dr. Kenneth M. Attie (Genentech, Inc., South San Francisco, CA), Dr. Bengt-Ake Bengtsson (University of Goteborg, Sahlgrenska Hospital, Goteborg, Sweden), Dr. Sandra L. Blethen (Genentech, Inc.), Dr. Werner Blum (Eli Lilly \& Co., Bad Homburg, Germany), Dr. Fergus Cameron (Royal Children's Hospital, Parkville, Australia), Dr. Jean Claude Carel (Hôpital St. Vincent de Paul, Paris, France), Dr. Lena Carlsson (Sahlgren Hospital, Goteborg, Sweden), Dr. John J. Chipman (Eli Lilly \& Co., Indianapolis, IN), Dr. Jens Sandahl Christiansen (Aarhus Kommunehospital, Aarhus, Denmark), Dr. Peter Clayton (Manchester Children's Hospital, Manchester, UK), Dr. David R. Clemmons (University of North Carolina, Chapel Hill, NC), Dr. Pinchas Cohen (Mattell Children's Hospital, Los Angeles, CA), Dr. Stenvert Drop (Sophia Children's Hospital, Rotterdam, The Netherlands), Dr. Kenji Fujieda (Hokkaido University School of Medicine, Sapporo, Japan), Dr. Ezio Ghigo (University of Turin, Turin, Italy), Dr. Raymond L. Hintz (Stanford University, Stanford, CA), Dr. Ken Ho (The Garvan Institute of Medical Research, Sydney, Australia), Dr. Mbelenge Mapoko Ilondo (Novo Nordisk A/S, Bagsvaerd, Denmark), Dr. Hector Jasper (CEDIE-CONICET, Buenos Aires, Argentina), Dr. Bernd Jesussek (Ferring Pharmaceuticals Ltd., Kiel, Germany), Dr. Anne-Marie Kappelgaard (Novo Nordisk A/S), Dr. Zvi Laron (Schneider Children's Medical Center of Israel, Petah Tiqva, Israel), Dr. Barbara M. Lippe (Pharmacia \& Upjohn, Inc., Los Angeles, CA), Dr. Saul Malozowski (FDA, Rockville, MD), Dr. Primus E. Mullis (Inselspital, Bern, Switzerland), Dr. Sabine de MuinckKeizer-Schrama (Sophia Kinderziekenhuls, Rotterdam, The Netherlands), Dr. Yoshikazu Nishi (Hiroshima Red Cross Hospital, Hiroshima, Japan), Dr. John S. Parks (Emory University School of Medicine, Atlanta, GA), Dr. Charlotte Phelps (Ferring Pharmaceuticals Ltd., Copenhagen, Denmark), Dr. Michael Ranke (University of Tubingen, Tubingen, Germany), Dr. Iain Robinson (National Institute for Medical Research, London, UK), Dr. Ron G. Rosenfeld (Oregon Health Sciences University, Portland, OR), Dr. Susan Rose (University of Tennessee, Memphis, TN), Dr. Paul Saenger (Albert Einstein College of Medicine, New York, NY), Dr. Guissepe Saggese (Universitá degli Studi di Pisa, Pisa, Italy), Dr.
The diagnosis and treatment of GH deficiency (GHD) during childhood and adolescence have been the subject of much controversy (1-3). To insure that patients are appropriately identified and treated, the GH Research Society (GRS) convened a workshop, on October 17-21 1999, in Eilat, Israel. The objectives of this workshop were to formulate consensus guidelines for the diagnosis and treatment of children and adolescents with GHD. The GRS invited clinicians and scientists with expertise in the field, representatives from industries involved in the manufacturing of recombinant $\mathrm{GH}$, and representatives from health authorities from a number of countries to attend the workshop. All of them contributed to the consensus guidelines as detailed below.

\section{Diagnosis of GHD in children}

The diagnosis of GHD in childhood is a multifaceted process requiring comprehensive clinical and auxological assessment, combined with biochemical tests of the GH-insulin-like growth factor (IGF) axis and radiological evaluation. GHD may present as an isolated problem or in combination with multiple pituitary hormone deficiency (MPHD). Each component of this process requires criteria, each of which will be considered below.

Clinical and auxological criteria. The evaluation for GHD in a short child, where short stature is defined as a height more than $2 \mathrm{sD}$ below the population mean, should not be initiated until other causes of growth failure, such as hypothyroidism, chronic systemic disease, Turner syndrome, or skeletal disorder, have been considered and appropriately excluded.

Key facts in the history and physical examination that may

Martin Savage (St. Bartholomew's Hospital School of Medicine, London, UK), Dr. Stephen Shalet (Christie Hospital, Manchester, UK), Dr. Pierre C. Sizonenko (Hopital Universitaire Cantonal des Enfants, Geneva, Switzerland), Dr. Christian Strasburger (Ludwig Maximilians Universitat, Munich, Germany), Dr. Katsuhiko Tachibana (Kanagawa Children's Center, Yokohama, Japan), Dr. Toshiaki Tanaka (National Children's Medical Research Center, Tokyo, Japan), Dr. Michael O. Thorner (University of Virginia Health Sciences Center, Charlottesville, VA), Dr. Kerstin Albertsson Wikland (Pediatric Growth Center, Goteborg, Sweden), and Dr. Zvi Zadik (Kaplan Hospital, Rehovot, Israel). 
indicate that GHD could be present include: 1 ) in the neonate: hypoglycemia, prolonged jaundice, microphallus, or traumatic delivery; 2) cranial irradiation; 3) head trauma or central nervous system infection; 4) consanguinity and/or an affected family member; and 5) craniofacial midline abnormalities.

It is recognized that short stature is often the only feature present. Criteria to initiate immediate investigation include: 1) severe short stature, defined as a height more than $3 \mathrm{SD}$ below the mean; 2) height more than 1.5 sD below the midparental height; 3 ) height more than 2 sD below the mean and a height velocity over 1 yr more than 1 sD below the mean for chronological age, or a decrease in height SD of more than 0.5 over 1 yr in children over 2 yr of age; 4 ) in the absence of short stature, a height velocity more than 2 sD below the mean over 1 yr or more than 1.5 sD sustained over 2 yr; this may occur in GHD, presenting in infancy, or in organic acquired GHD; 5) signs indicative of an intracranial lesion; 6) signs of MPHD; and 7) neonatal symptoms and signs of GHD.

It should be noted that the interpretation of growth data requires the most recent relevant population standards available. Where possible, these standards should be updated every 10-20 yr, dependent on the population secular trend. Growth data should be expressed as SD scores rather than as percentiles. To correctly evaluate height velocity, there is a need for longitudinal velocity standards. Biological markers outside the GH-IGF axis, such as body composition, bone density, and bone markers are presently not discriminatory for the diagnosis of GHD.

With the increasing use of magnetic resonance imaging (MRI), it is recognized that an incidental MRI abnormality within the hypothalamic-pituitary region may be detected. This requires clinical evaluation of the child and possibly growth surveillance. In the appropriate clinical context, an ophthalmological examination may be needed.

Evaluation for genetic disorders. The precise etiology of genetic disorders of GHD and MPHD (e.g. PROP1 and POU1F1 mutations) are being increasingly recognized. Pointers to such conditions include: 1) early onset of growth failure, 2) positive family history and possible consanguinity, 3) height more than 3 sD below the mean, and 4) extremely low GH response to provocation tests, including GHRH, and very low IGF-I and IGF-binding protein-3 (IGFBP-3) levels.

Tests for genetic mutations are presently only available in research laboratories. It is preferable that these tests become more widely available. Efforts to bank DNA should be made, respecting ethical and legal considerations.

Radiological evaluation. Bone age estimated from an x-ray of the left wrist and hand should be undertaken as part of the routine evaluation of children with growth failure over $1 \mathrm{yr}$ of age and should be read by an experienced person. In infants less than $1 \mathrm{yr}$, bone age estimated from $x$-rays of the knee and ankle may be useful.

Central nervous system imaging by MRI or computerized tomography (CT) is required in those with known or suspected intracranial tumors, optic nerve hypoplasia/septooptic dysplasia or other structural or developmental anom- aly. In confirmed isolated GHD or MPHD with or without genetic defects, the following features should be recorded from the MRI (ideally in 2-mm slices with and without contrast): pituitary height and/or volume, anatomy of the stalk, and position of the posterior pituitary. However, it is recognized that more normative morphological data are required to improve the quality of this assessment. The resolution of the hypothalamic-pituitary region is inferior on CT scanning, but the latter is useful for tumors and bone abnormalities. Intracranial calcification, as often seen in craniopharyngioma, can be detected on skull radiograms.

Biochemical assessment of GHD. Assay considerations: At present, there is a wide range of assays available to measure GH, IGF-I, and IGFBP-3. To improve standardization, it is recommended that the $\mathrm{GH}$ reference preparation should be a recombinant 22-kDa human GH (hGH; presently 88/624, which has been assigned a potency of $3 \mathrm{IU}=1 \mathrm{mg}$ ). There is currently no acceptable IGF-I reference preparation, and a WHO preparation based on recombinant human IGF-I is needed. The use of appropriate reference preparations should also be applied to any other peptide or binding protein assay.

When reporting assay data, a clear statement of methodology is required. The clinician, using any assay, should be aware of its methodology, its limitations, and its performance in the diagnosis of GHD. An assay that measures 22-kDa hGH, using monoclonal antibodies, is recommended. The immunofunctional $\mathrm{GH}$ assay requires further evaluation.

GH provocation tests and IGF-I/IGFBP-3 measurements: A limited number of provocative agents should be used after an overnight fast in a well standardized protocol. These include arginine, clonidine, glucagon, insulin, and L-dopa. These tests should be monitored carefully by an experienced team. Great care should be exercised in using insulin or glucagon in a young child. Limited reference data for each of these GH tests exist, and more data in normal children are desirable within ethical guidelines.

In a child with clinical criteria for GHD, a peak GH concentration below $10 \mu \mathrm{g} / \mathrm{L}$ has traditionally been used to support the diagnosis. This value needs to be revised when using newer monoclonal-based assays and recombinant hGH reference preparations. There exists a continuum of GH secretion that ranges from moderate GHD to severe GHD, as seen in congenital or acquired MPHD. However, it also is recognized that overlap exists in peak GH concentrations between normal children and those with GHD. For IGF-I and IGFBP-3, reference ranges, standardized for age and sex, are mandatory. Values below a cut-off less than -2 sD for IGF-I and/or IGFBP-3 strongly suggest an abnormality in the GH axis if other causes of low IGF have been excluded. Nevertheless, in GHD, values of IGF-I and IGFBP-3 within the normal range can occur. In the absence of a gold standard, it is therefore important that the clinician integrates all available data (clinical, auxological, radiological, and biochemical) when making a diagnosis.

Sex steroid priming. The difficulty of diagnosing GHD during the immediate peripubertal period is acknowledged, as low 
GH levels in provocation tests frequently occur. At the present time there is no consensus on the use of priming with sex steroids before GH tests.

Testing in the neonate. A GH level should always be measured in the presence of neonatal hypoglycemia in the absence of a metabolic disorder. A random GH measurement in a polyclonal RIA of less than $20 \mu \mathrm{g} / \mathrm{L}$ would suggest GHD in the newborn. An IGFBP-3 measurement is of value for the diagnosis of GHD in infancy.

Other tests of the GH axis. It is considered that urinary GH, serum IGF-II, IGFBP-2, and acid-labile subunit levels and GH secretagogues (as provocative agents) are not diagnostic by themselves, but may be useful in combination with other tests. The combination of GHRH and arginine may be of value in the diagnosis of GHD, provided appropriate cut-off limits are applied. It has high specificity (normal results in normally-growing children).

The evaluation of spontaneous GH secretion over time (12 or $24 \mathrm{~h}$ ), generated from a standardized protocol with appropriate reference data, can be considered when $\mathrm{GH}$ and IGF data conflict, such as normal GH and low IGF-I. Nevertheless, it is recognized that neurosecretory dysfunction (in the absence of a history of cranial irradiation) is uncommon.

Experience with the IGF-I/IGFBP-3 generation test in the diagnosis of GHD is insufficient. However, the generation test is required for the diagnosis of GH insensitivity (resistance). GH bioinactivity is an exceedingly rare diagnosis.

Confounding factors. It is very important to recognize the influence of factors such as nutritional status, concomitant medication (e.g. glucocorticoids, psychotropic drugs, etc.), and psychosocial conditions on growth and the GH-IGF axis. The clinician should be constantly vigilant for signs that may suggest the latter condition.

Process of evaluation of the GH-IGF axis. In a child with slow growth, whose history and auxology suggest GHD, testing for GH/IGF-I deficiency requires IGF-I/IGFBP-3 levels and GH provocation tests after hypothyroidism has been excluded. In suspected isolated GHD, two GH provocation tests (sequential or on separate days) are required. In those with defined central nervous system pathology, history of irradiation, MPHD, or a genetic defect, one GH test will suffice. In addition, an evaluation of other pituitary function is required. In patients who have had cranial irradiation or malformations of the hypothalamic-pituitary unit, GHD may evolve over years, and its diagnosis may require repeat testing of the GH-IGF axis.

It is recognized, however, that some patients with auxology suggestive of GHD may have IGF-I and/or IGFBP-3 levels below the normal range on repeated tests, but GH responses in provocation tests above the cut-off level. These children are not classically GH deficient, but may have an abnormality of the GH/IGF axis and, after the exclusion of systemic disorders affecting the synthesis or action of IGF-I, could be considered for GH treatment.

A MRI (or CT scan) of the brain with particular attention to the hypothalamic-pituitary region should be carried out in any child diagnosed as having GHD.
Conclusion. The diagnosis of severe GHD is usually straightforward, as there are well defined clinical, auxological, biochemical, and radiological abnormalities. However, the diagnosis of moderate GHD can be associated with normal values within the IGF axis and a normal MRI. It is very important that the response to $\mathrm{GH}$ treatment be carefully reviewed, particularly in those patients with moderate GHD.

\section{Treatment of GHD in children}

Patients with proven GHD should be treated with recombinant hGH as soon as possible after the diagnosis is made. The primary objectives of the therapy of GHD are normalization of height during childhood and attainment of normal adult height. Normally growing patients with craniopharyngioma and GHD should be considered for therapy with $\mathrm{GH}$ for metabolic and body composition benefits and for enhancement of pubertal growth. Insufficient data regarding the utility of GHRH, GH secretagogues, and depot GH are currently available to formulate recommendations regarding their use in GHD.

Dosing of GH. GH should be administered sc in the evening on a daily basis, and the dosage of GH should be expressed in milligrams (or micrograms) per $\mathrm{kg} /$ day, although consideration should be given to dosing in micrograms per $\mathrm{m}^{2} /$ day in patients with obesity. GH is routinely used in the range of $25-50 \mu \mathrm{g} / \mathrm{kg} /$ day. A dose-response relationship in terms of height velocity in the first $2 \mathrm{yr}$ of treatment has been clearly demonstrated within this range. Under special circumstances, higher doses may be required. Prediction models of growth response might be useful for determination of the optimal individual dose and are currently being investigated, but need further evaluation.

Monitoring GH therapy. The routine follow-up of pediatric GHD patients should be performed by a pediatric endocrinologist in partnership with the pediatrician or primary care physician and should be conducted on a 3- to 6-month basis. The determination of the growth response to GH treatment is the single most important parameter in the monitoring of the child with GHD. Increase in height and change in height velocity are useful in clinical practice to assess the response to GH. For comparative purposes, data should be expressed as the increase in (or $\delta$ ) height SD per yr. For assurance of compliance and safety, monitoring of serum IGF-I and IGFBP-3 levels is useful, although they do not always correlate well with the growth response. There are insufficient data to support the use of serum leptin or bone markers in monitoring GH therapy. The routine monitoring of GH antibodies has no value in GHD management. Lipid profiles and fasting insulin levels are not routinely measured in the child receiving GH therapy.

Factors affecting the response to GH. Every effort should be made to diagnose and treat children at the youngest possible age. It is very important to maximize height with GH therapy before the onset of puberty. If this is achieved, then modulation of the GH dose during puberty may not be necessary. Treatment of children entering puberty at an inadequate height, using GH dose escalation or combined GH and GnRH agonist therapy, is presently being evaluated. In the MPHD 
patient in whom puberty does not occur spontaneously, puberty should be initiated at the appropriate time after discussion with the patient. The possible role of gender and body composition in the response to GH in GHD children requires further investigation.

Management of MPHD. Patients with suspected or proven multiple pituitary hormone deficiencies should be managed similarly to patients with isolated GHD; however, attention should be given to correct clinical recognition, treatment, and monitoring of additional hormonal deficiencies $\left(\mathrm{T}_{4}\right.$, cortisol, sex steroids, and antidiuretic hormone). In the patient with an initial diagnosis of isolated GHD, particularly those with ectopic posterior pituitary or other developmental abnormalities, the clinician should be alert to the risk of the development of MPHD.

Safety issues. Treatment with GH may unmask underlying hypothyroidism. Significant side-effects of GH treatment in children are very rare. These include benign intracranial hypertension, prepubertal gynecomastia, arthralgia, and edema. A careful history and physical examination are adequate to identify their presence. Management of these sideeffects may include either transient reduction of dosage or temporary discontinuation of GH. In the absence of other risk factors, there is no evidence that the risk of leukemia, brain tumor recurrence, slipped capital femoral epiphysis, or diabetes is increased in recipients of long-term GH treatment. Tumor survivors receiving GH should be followed in conjunction with an oncologist and a neurosurgeon when appropriate. There is no evidence that GH replacement needs to be discontinued during intercurrent illness.

Transition to adult management. GHD may or may not persist into adult life. GH has major metabolic actions, which are important for body composition and health in adults as well as in children. After the attainment of final height, retesting of the GH-IGF axis, using the adult GHD diagnostic criteria, as defined by the GRS Consensus Workshop on adult GHD in 1997 at Port Stephens (4), should be undertaken by the pediatric endocrinologist using standard GH stimulation tests after an appropriate interval of 1-3 months off GH therapy. In places where an insulin tolerance test is mandatory for the patient to qualify for further GH therapy, this test should be performed. At the time of retesting, other pituitary hormones and IGF-I should also be measured. The opportunity should be taken to assess body composition, bone mineral density, and fasting lipids and insulin before and after discontinuation of GH therapy. Quality of life should be assessed using validated age- and disease-appropriate instruments, which at this time require development. Patients with severe long-standing MPHD, those with genetic defects, and those with severe organic GHD can be excluded from $\mathrm{GH}$ retesting. When the diagnosis of adult GHD is established, continuation of GH therapy is recommended. Caution should be exercised when considering the decision of continuing GH therapy in conditions where there is a known risk of diabetes or malignancy. The transition to adult GH replacement should be arranged as a close collaboration between the pediatric and adult endocrinologists, who should discuss the reinitiation of treatment with the patient.

Conclusion. The approach to the diagnosis and treatment of GHD in children has been evolving over the last several decades. The current consensus document represents the results of intensive review and discussion of thousands of published reports and an incalculable amount of personal experience by experts all over the world. Clearly, many additional evidence-based clinical studies involving the diagnosis and treatment of GHD will continue to be performed and will undoubtedly modify the approach to this important issue. It will be the goal of the GRS as well as the pediatric endocrine societies and bodies, which have endorsed this document, to amend and revise this statement in coming years.

\section{References}

1. Drug and Therapeutics Committee of the Lawson Wilkins Pediatric Endocrine Society. 1995 Guidelines for the use of growth hormone in children with short stature: a report by the Drug and Therapeutics Committee of the Lawson Wilkins Pediatric Endocrine Society. J Pediatr. 127:857-867.

2. American Academy of Pediatrics. 1997 Considerations related to the use of recombinant human growth hormone in children. Pediatrics. 99:122-129.

3. Saggese G, Ranke MB, Saenger P, Rosenfeld RG, Tanaka T, Chaussain JL, Savage MO. 1998 Diagnosis and treatment of growth hormone deficiency in children and adolescents; towards a consensus. Horm Res. 50:320-340.

4. Growth Hormone Research Society Workshop on Adult Growth Hormone Deficiency. 1998 Consensus guidelines for the diagnosis, treatment of adults with growth hormone deficiency: summary statement of the Growth Hormone Research Society Workshop on Adult Growth Hormone Deficiency. J Clin Endocrinol Metab. 93:379-381. 